\title{
View variations Effect in Human Gait Recognition using Sub-Window Extraction Algorithm
}

\author{
Gyan C. Shivhare, \\ Maharana Pratap College of Technology \\ Gwalior, India
}

\author{
Unmukh Datta \\ Maharana Pratap College of Technology \\ Gwalior, India
}

\begin{abstract}
In this paper, the view variations effect in human gait recognition using sub-window extraction algorithm is proposed. Here different variation is created based on the walking people in three different angles (i.e. $0^{\circ}, 45^{\circ}$ and $90^{\circ}$ )with respect to particular line. Our proposed method works on two different phases: Extraction phase and Recognition Phase. In first phase, gait images, captured from different angles, are enhanced using clipping, filtering and histogram equalization. Then apply proposed sub window extraction algorithm on enhanced gait images and gathered different features like person length, leg angle, leg length, hand length etc. Finally apply back propagation algorithm for the recognition of gait images. Experiments are carried out using different datasets.
\end{abstract}

\section{Keywords}

Back propagation algorithm, BPA, Gait Recognition, Neural Network, Sub-window extraction

\section{INTRODUCTION}

Human recognition is an important task for authentication process and used in different applications like access control, surveillance, etc. To distinguish different persons having different walking manner is a natural task people perform every day.Recognition through gait is special interest because itcan identify people from some distance, without cooperation of users.The main factor that effects the human identification is the view variation problem, because view ofthe person can be change, when peoples are beingrecognition through gait recognition system $[1,7,9]$. view invariant gait recognition algorithmdescribed in $[2,3,6]$. In most recognition approaches [5,6,10], recognition features are extracted from silhouette images. Modified ICA based efficient gait recognition for human identification is described in [4].

For human identification, Gait is one of well recognized biometrics that has been widely used. Biometric authentication is an automated human recognition methodbased on their behavioral and physiological characteristic. Physiological biometricsconsists of face recognition, fingerprints recognition, iris-scans and hand scans and behavioral, like keystroke-scan and speech patterns recognition.

Fig 1 shows the process of gait detection.
Fig 1 shows process of gait detection (a) background

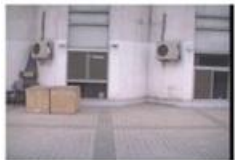

(a)

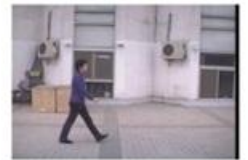

(b)

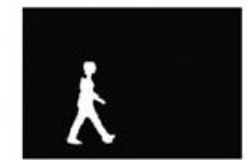

(c) image (b) original image (c) silhouette image

The remainders of this paper are organized as follows. Section 2 explains the preprocessing strategy of human gait images. Section 3 explains Back propagation algorithm. Section 4 explains the proposed algorithm and experiments are conducted on different datasets in Section 5. Finally, in Section 6, Section 7 and Section 8 give the conclusion, acknowledgement and provides the references used in this work respectively.

\section{PREPROCESSING}

To identify the effect of view angle on human gait recognition performance, a multi view gait database is needed. In this paper, databases are collected from internet via http://www.gait.ecs.soton.ac.uk/database/

Here three different view angles of walking path person is shown in fig 2 .

Human gait images, collected from different sources, may be of poor brightness and contrast due to poor lighting condition. So histogram equalization is used for enhancing the brightness and contrast of the gait image.Filteringand clipping techniques are used to remove noise and unnecessary background data.

\section{BACK PROPAGATION ALGORITHM}

Back propagation is the common method for teaching artificial neural network (ANN). It was created by generalizing the Windrow- Hoff learning rule to multiple-layer networks and nonlinear differentiable transfer functions. Back propagation algorithm is used for the recognition purpose. In this, initially all gait features (mouth, eyes and nose) are collected from sub windows, which are extracted from sub window extraction algorithms. Then these features are normalized between $[0,1]$ by dividing each values of the image vector to the maximum value from the vector. These normalized values are assigned to input neurons. Initial weights and bias values are assigned randomly between $[-1,1]$. Output from each hidden neuron is calculated 
using sigmoid activation function. Mean square error (MSE) is calculated at the output neuron and then back propagates the error and find the delta values at output neurons. With these value, update weights and bias values and again the same procedure is repeated until target output is not achieved.

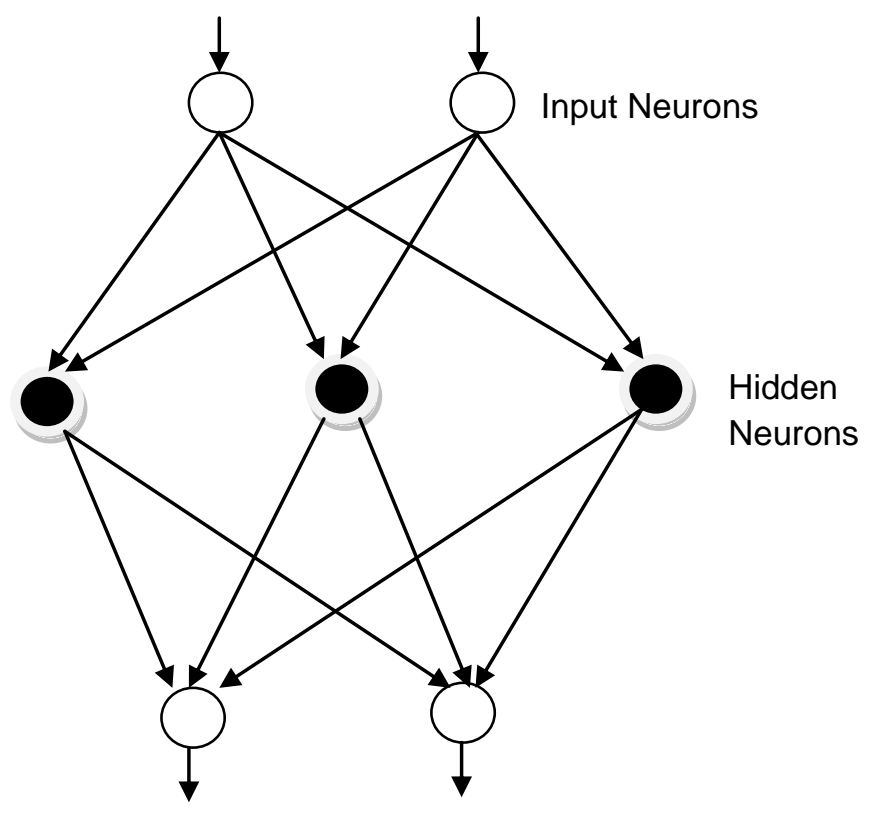

\section{PROPOSED METHODOLOGY}

This proposed method works on two phases, in the first phase; gait database is collected from different sources like internet, manually from camera. After that, gait images are enhanced using filtering, contrast/brightness, noise removal and clipping for extracting unnecessary background. Now, proposed subwindow extraction algorithm is used for extracting the sub-window from the silhouette image.in this algorithm, firstly convert the original gait image into silhouette image. Then load the silhouette image and search the upper-left, upper-right, lower-left and lower-right pixels for each row and each column. With the help of these pixels, extract sub-window from the silhouette image. Now calculate the mean of the extracted sub-window. If the mean of the extracted window is zero then extracted sub-window will be considered as a background image and skipped. else will be considered as sub-window. The given algorithm is applied at each row and column for extracting the sub-window.

In the second phase, back propagation algorithm is used for the recognition for getting the efficiency of the proposed method.

\section{Output Neurons}

Fig 3: 2-layered feed forward network

\section{Proposed Sub-Window extraction algorithm}

Step 1: Convert the original enhanced gait image into silhouette image

Step 2: Load the silhouette image

Step 3: Search the upper-left, upper-right, lower-left and lower-right pixels for each row and each column

Step 4: From silhouette image, extract sub-window

Step 5: Calculate the mean of the extracted sub-window using following equation (1)-

$$
\bar{X}=\frac{1}{N} \sum_{1}^{N} X_{i}
$$

Step 6: if the mean of the extracted window is zero then sub-window considered as a background image and skipped. GOTO step 3 for extracting the next sub window

Step 7: else, from silhouette image, extract the corresponding sub-window 

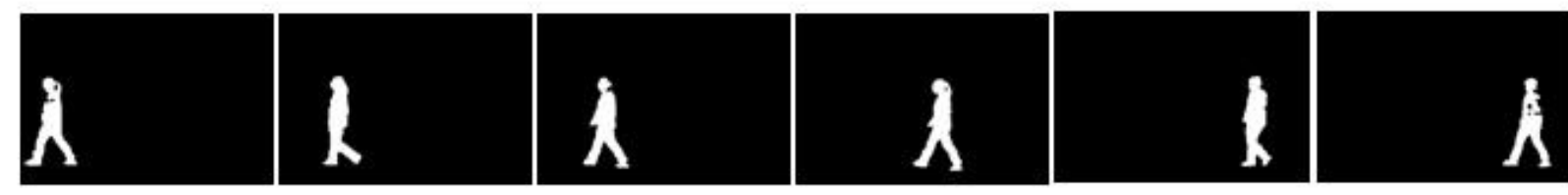

Moving Gait Images at $0^{\circ}$
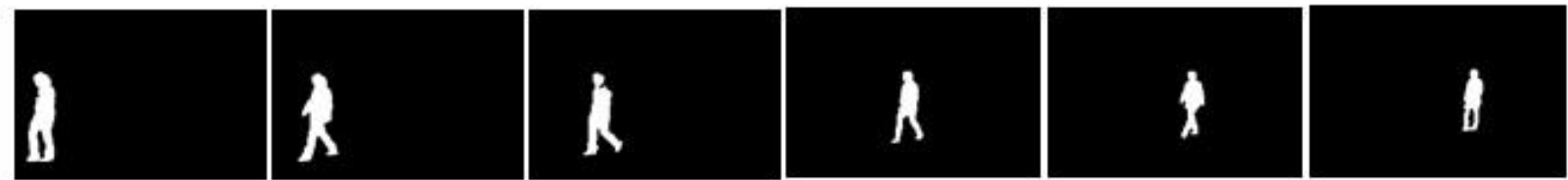

Moving Gait Images at $45^{\circ}$
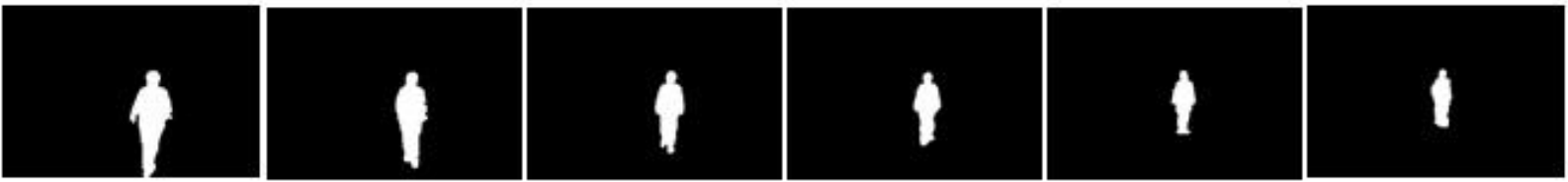

Moving Gait Images at $90^{\circ}$

Fig 2 : Shows the moving gait images at different angles (i.e. $0^{0}, 45^{\circ}, 90^{\circ}$ )

\section{EXPERIMENTS AND RESULTS}

All the algorithms are implemented in MATLAB 7.6.8 and executed on the Pentium-IV, 3.00GHz CPU with 4 GB RAM.Experimental dataset consists of mixed male and female images having 70 different images of each person and there are 50 persons in the dataset. These images at different angles, like $0^{\circ}, 45^{\circ}, 90^{\circ}$, are shown in fig 2 . Here, original data set grouped into different data sets as shown in table 3 below and each group is partitioned into three subsets viz. training set, testing set and validation set.Table 3 shows the dataset partition having different percentage of training, testing and validation gait images. Table 4 and table 5 shows that recognition training, testing and validation accuracy over different data sets and different number of hidden nodes.
Table 3: Shows that different dataset partition groups

\begin{tabular}{|c|c|c|c|}
\hline $\begin{array}{c}\text { Data Set } \\
\text { (Group) } \\
\text { Name }\end{array}$ & $\begin{array}{c}\text { Training } \\
\text { (\% age) }\end{array}$ & $\begin{array}{c}\text { Testing } \\
\text { (\% age) }\end{array}$ & $\begin{array}{c}\text { Validation } \\
\text { (\% age) }\end{array}$ \\
\hline $\begin{array}{c}\text { Data set } 1 \\
\text { (DS1) }\end{array}$ & 50 & 25 & 25 \\
\hline $\begin{array}{c}\text { Data set } 2 \\
\text { (DS2) }\end{array}$ & 60 & 15 & 20 \\
\hline $\begin{array}{c}\text { Data set 3 } \\
\text { (DS3) }\end{array}$ & 70 & 10 & 10 \\
\hline $\begin{array}{c}\text { Data set } 4 \\
\text { (DS4) }\end{array}$ & 80 & 5 & 5 \\
\hline $\begin{array}{c}\text { Data set 5 } \\
\text { (DS5) }\end{array}$ & 90 & & \\
\hline \multicolumn{2}{|l}{}
\end{tabular}


Table 4: Data set results based on different partitions

\begin{tabular}{|c|c|c|c|}
\hline \multirow{2}{*}{$\begin{array}{c}\text { No. of } \\
\text { Hidden } \\
\text { neurons }\end{array}$} & \multicolumn{3}{|c|}{$\begin{array}{c}\text { Back propagation } \\
\text { (Accuracy) }\end{array}$} \\
\cline { 2 - 4 } & $\begin{array}{c}\text { Training } \\
(\%)\end{array}$ & $\begin{array}{c}\text { Testing } \\
(\%)\end{array}$ & $\begin{array}{c}\text { Validation } \\
(\%)\end{array}$ \\
\hline 10 & 93.16 & 90.77 & 87.33 \\
\hline 20 & 96.41 & 96.12 & 94.62 \\
\hline 30 & 96.31 & 98.33 & 98.33 \\
\hline 40 & 96.12 & 94.87 & 96.91 \\
\hline
\end{tabular}

Table 5: shows that the accuracy at different number of hidden nodes

\begin{tabular}{|c|c|c|c|}
\hline $\begin{array}{c}\text { Data Set } \\
\text { Groups }\end{array}$ & $\begin{array}{c}\text { Training } \\
\text { Accuracy } \\
(\boldsymbol{\%})\end{array}$ & $\begin{array}{c}\text { Testing } \\
\text { Accuracy } \\
(\boldsymbol{\%})\end{array}$ & $\begin{array}{c}\text { Validation } \\
\text { Accuracy } \\
(\%)\end{array}$ \\
\hline DS1 & 94.51 & 90.39 & 92.72 \\
\hline DS2 & 97.54 & 94.82 & 91.33 \\
\hline DS3 & 98.01 & 92.11 & 96.23 \\
\hline DS4 & 96.74 & 92.65 & 94.29 \\
\hline DS5 & 93.55 & 90.05 & 91.49 \\
\hline
\end{tabular}

\section{CONCLUSION}

The main objective of this paper is to propose an algorithm that improves the recognition time and accuracy. so for getting better result and improves recognition time, we have proposed a sub windows extraction algorithm that will divides the gait image into different sub windows and by using these sub windows, find out the gait features and trained with back propagation algorithm, as a recognition algorithm, for getting the recognition accuracy. Advantage of this algorithm is that it reduce the number of subwindows.our proposed algorithm is tested using three different types of view angles of human gait images. Finally the proposed results using this algorithm is better than other algorithm in terms of accuracy and recognition time. Further research is to check the proposed algorithm on real life images.

\section{ACKNOWLEDGEMENTS}

This work is supported by Maharana Pratap College of Technology, Gwalior - India

\section{REFERENCES}

[1] Sarkar, S., Phillips, PJ., Liu, Z., Vega, I.R., Grother, P., Bowyer,K.W., "The human ID gait challenge problem: data sets,performance, and analysis," Proceeding of the IEEE Transactionson Pattern Analysis and Machine Intelligence, vol.27, no.2,pp.162-177, 2005.

[2] Shiqi $\mathrm{Yu}^{\prime}$ Daoliang Tan, and Tieniu Tan, "Modelling the Effect ofView Variation on Appearance-based Gait Recognition",Proceeding of the IEEE Asian Conference on Computer Vision(ACCV06). Hyderabad, India. Jan. 2006.

[3] Kale, A.; Chowdhury, A.K.R.; Chellappa, R.; , "Towards a viewinvariant gait recognition algorithm, " Proceedings. IEEEConference on Advanced Video and Signal Based Surveillance,2003., vol., no., pp. 143- 150, 2 1-22 July 2003.

[4] M. Pushpa Rani! and G.Ammugam,"An Efficient Gait ecognitionSystem for Human Identification using Modified ICA.",International Journal of Computer Science \& InformationTechnology (IJIST), Vol 2, No. 1, Feb 2010.

[5] J. P. Foster, M. S. Nixon, and A. Prudel-Bennett. Automaticgait recognition using area-based metrics. Pattern Recognition Letters, 24(14):2489-2497, 2001.

[6] Shiqi Yu; Daoliang Tan; Tieniu Tan; , "A Framework forEvaluating the Effect of View Angle, Clothing and CarryingCondition on Gait Recognition, " Pattern Recognition, 2006. ICPR2006. 18th International Conference on , vol.4, no., pp.441-444.

[7] Ishikawa, E; Karungaru, S; Terada, K.; "Gait features extractionusing image processing", Frontiers of Computer Vision (FCS),2011 17"' Korea-Japan joint Workshop, pp. 1-6, 9-11 feb, 2011.

[8] A. Kale, N. Cuntoor, B. Yegnanarayana, A.N Rajagopalan,and R. Chellappa. Gait analysis for human identification.In Proceedings of the 3rd International conference on Audioand Video Based Person Authentication, 2003.

[9] Uan Wang, Shiqi Yu, Yunhong Wang and Tieniu Tan. GaitRecognition Based on Fusion of Multiivew Gait Sequences. InProc. of the International Conference on Biometrics 2006. Pages605-611. Hong Kong, China. Jan. 2006.

[10] P.J. Phillips, S. Sarkar, I. Robledo, P. Grother, andK. Bowyer. The gait identification challenge problem: Datasets and baseline algorithm. In International Conference onPattern Recognition, 2002. 